\title{
Vom Pflanzenreich ins Schlummerland
}

\author{
Verschiedene Heilpflanzen können bei SCHLAFPROBLEMEN helfen. Doch wann führt \\ welche Pflanze den Schlafsuchenden ans ersehnte Ziel? \\ Cornelia Stern
}

\begin{abstract}
OB BEVORSTEHENDE ABSCHLUSSPRÜFUNG, die roten Zahlen auf dem Kontoauszug oder Ängste und Unruhe infolge einer Depression: Es gibt vielfältige Ursachen und Auslöser von Schlafstörungen. Wie gut, dass Mutter Natur uns unterschiedlichste Schlafpflanzen schenkt. Wir müssen nur genau hinschauen, um die richtige für die vom Schlafmangel zermürbten Patienten zu finden. Die Signaturenlehre ist hierbei ein guter Wegweiser. Mit ihr erweitern wir unseren
\end{abstract}

Blick für die individuelle Symptomatik und Situation jedes Patienten, für den Charakter einer jeden Schlafstörung und dafür, welcher Schatz aus dem großen Pflanzenreich welchem Schlaflosen den Weg ins Schlummerland ebnen kann.

\section{Merke: Auch wenn dieser Artikel die Wirkweise dieser bewährten Heilpflanzen aus Sicht der Signa- turenlehre beschreibt, haben alle mit Ausnahme des Kalifornischen}

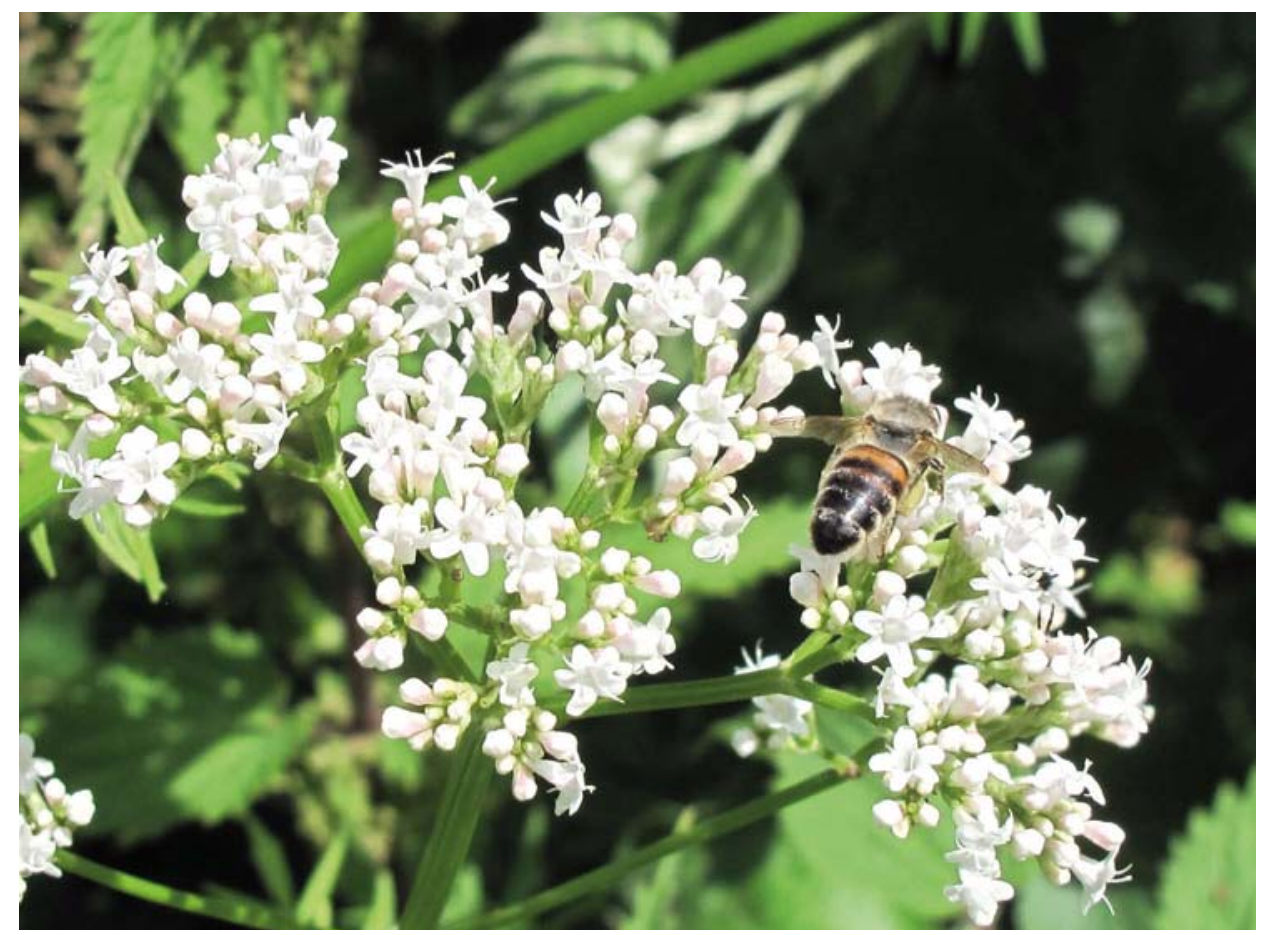

Abb. 1 Baldrian: Die lichten Blütenstände verströmen einen lieblichen Duft. Das bei älteren Pflanzen stark verwachsene Wurzelgeflecht riecht erdig-modrig. Baldrian schenkt Leichtigkeit und Erdung zugleich.

\begin{abstract}
Mohns eine HMPC-Monografie erhalten mit der Indikation nervöse Unruhezustände und/oder Einschlafstörungen.
\end{abstract}

\section{Einschlaf- und/oder Durch- schlafstörungen?}

Wichtig für die Verordnung ist zu unterscheiden, ob der Patient nicht ein- und/ oder nicht durchschlafen kann. Denn Durchschlafstörungen sind mit Pflanzenkraft wesentlich schwieriger in den Griff zu bekommen als Einschlafprobleme. Wieso ist das so? Das hat zum einen damit zu tun, dass pflanzliche Präparate keine Wirkung über die ganze Nacht erzielen können - sie werden im Körper zu schnell abgebaut. Zum anderen verbergen sich hinter Durchschlafproblemen oft psychische Belastungen. Oder es liegt eine organische Belastung vor, und das Organ meldet sich immer zur gleichen Zeit und weckt den Schlafenden. Zum Beispiel hat die Leber gemäß der chinesischen Organuhr ihren Aktivitätshöhepunkt zwischen 1 und $3 \mathrm{Uhr}$ morgens. Wacht ein Patient immer zu dieser Zeit auf, nützen meist alle Schlafpflanzen nichts. Dann benötigt der Patient mit großer Wahrscheinlichkeit Leberpflanzen wie Mariendistel (Früchte), Schafgarbe (Kraut oder Blüten) oder Artischocke (Blätter). Zusammenfassend lässt sich sagen, dass Durchschlafstörungen schwieriger als Einschlafstörungen mit phytotherapeutischen Präparaten zu behandeln sind und das Resultat oft nicht sehr befriedigend ist. 
Entscheidet sich der Behandelnde nach der umfangreichen Anamnese und Untersuchung für eine Verordnung von Schlafpflanzen, gilt es, die Unterschiede der Pflanzen unter die Lupe zu nehmen. Die Signaturen einer Pflanze geben unter anderem Hinweise auf ihre spezifischen Wirkungen und Anwendungsgebiete. So lassen sich für jeden Schlafsuchenden die richtigen Heilpflanzen auswählen.

\section{Baldrian (Valeriana officinalis)}

Wenn man von Schlafproblemen und geeigneten Heilpflanzen spricht, dann denken wohl die meisten an den Baldrian. Baldrian, die Schlafpflanze Nummer 1 unzählige Präparate mit zugehöriger Werbung sprechen für sich. Und doch ist der Baldrian für viele Patienten eben nicht die richtige Pflanze, um Schlafstörungen zu therapieren. Zwar wird er gerade in Alters- und Pflegeheimen aufgrund seiner großen Popularität bevorzugt verordnet, doch sind bei alten und pflegebedürftigen Patienten andere Pflanzen oft sehr viel geeigneter (siehe „Kalifornischer Mohn“).

\section{Signatur: Leichtigkeit und Erdung zugleich}

Schaut man sich die Baldrianpflanze an, sieht man oberirdisch lange Stiele mit lichten Blütenständen. Alles wirkt ätherisch leicht und streckt sich dem Himmel zu. Die gefiederten Blätter sind regelmäßig und rhythmisch aufgebaut. Das Wurzelwerk älterer Pflanzen hingegen ist im Vergleich zu der eher filigranen oberirdischen Pflanze ein wild durcheinander wachsendes Geflecht von erstaunlicher Größe.

Schnuppert man an den weiß-rosa Blüten, strömt einem der lilienartige Duft entgegen. Ganz anders riechen die Wurzeln: Ihr typischer Geruch erinnert an einen modrig-feuchten Keller. Leider riechen auch die Teezubereitung und die Tinktur aus den Wurzeln so. Der Geruch scheidet die Geister: Man mag ihn oder hasst ihn.

Der Baldrian ist also eine Pflanze, die sich oben dem Leichten, dem Himmel entgegenstreckt und wohlriechende

\title{
KURZ GEFASST
}

\author{
1 Die Pflanzenheilkunde bietet verschiedene Heilpflanzen für den \\ Einsatz bei Schlafstörungen: unter anderem Baldrian, Kaliforni- \\ schen Mohn, Hafer, Passionsblume und Hopfen. \\ 2 Nicht jede Schlafpflanze ist für jeden Patienten gleich gut geeig- \\ net. Die Signaturenlehre hilft, die richtige je nach Ursache, indivi- \\ dueller Symptomatik und Situation des Patienten auszusuchen. \\ 3 Die meisten der vorgestellten Schlafpflanzen sind auch von der \\ Rationalen Phytotherapie mit den Indikationen Unruhezustände \\ und/oder Schlafstörungen anerkannt.
}

ätherische Öle ausscheidet. Unten findet man ein erdig riechendes Wurzelgeflecht, das die Pflanze gut im Boden verankert, sozusagen erdet. Und dazwischen das rhythmisch aufgebaute Blattwerk. Was sagt uns das?

\section{Wirkung: Schlafanstoß für die} Nacht, Fokussierung für den Tag Wer Baldrian-Fertigarzneimittel genauer unter die Lupe nimmt, findet im Beipackzettel den Hinweis, dass man den Baldrian nachts bei Schlafstörungen einnehmen kann und tagsüber als Mittel bei Nervosität und Unruhe. Denn das Besondere am Baldrian ist, dass er tagsüber eingesetzt nicht müde macht. Im Gegenteil: Er steigert die Konzentration, macht wach, lenkt den Blick auf das Wesentliche und zentriert.

Erklärbar ist das Phänomen damit, dass der Baldrian nicht in erster Linie schlaffördernd wirkt, sondern rhythmisierend, also den verlorenen Rhythmus von Tag und Nacht zurückbringt. Wie oft machen wir die Nacht zum Tag, besonders in stressigen Zeiten, in denen wir bis tief in die Nacht arbeiten - Kunstlicht sei Dank. Der Baldrian erinnert den Körper sozusagen daran, dass der Tag zum Arbeiten da ist und die Nacht zum Erholen und Verarbeiten des Erlebten. Darauf weist auch die Signatur der rhythmisch aufgebauten Blätter hin. Der Baldrian ist also die ideale Schlafpflanze für Menschen, die den Tag-Nacht-Rhythmus verloren haben, die sich im Hamsterrad des Stresses unaufhörlich drehen und dann nachts vor lauter Gedankenkarussell auch keinen Schlaf finden. Das erklärt auch, wieso der Baldrian nicht die ideale Pflanze für die schlaflosen Bewohner in Alters- und Pflegeheimen ist. Denn deren Problem ist nicht Stress oder ein verlorengegangener Tag-Nacht-Rhythmus, sondern zum Beispiel fehlende Geborgenheit (siehe „Kalifornischer Mohn“).

\section{Baldrian-Präparate (Auswahl)}

- getrocknete Baldrianwurzeln für die Teezubereitung

- Valeriana Urtinktur (Fa. Ceres): 1- bis 3-mal tgl. 2-5 Tr. beziehungsweise vor dem Schlafengehen 3-7 Tr. in etwas Wasser einnehmen

- Valeriana comp. Urtinktur (Fa. Ceres): Kombination mit Hopfen, Melisse und Petasites D6, 1- bis 3-mal tgl. 2-5 Tr. beziehungsweise vor dem Schlafengehen 3-7 Tr. in etwas Wasser einnehmen

- Baldriparan ${ }^{\circledR}$ zur Beruhigung (Fa. Pfizer): Kombination mit Hopfen und Melisse, $3 \times \operatorname{tgl}$. 2 Tbl. einnehmen - Baldriparan ${ }^{\circledR}$ stark für die Nacht (Fa. Pfizer): vor dem Schlafengehen 1 Tbl. einnehmen

- Valeriana Hevert Beruhigungsdragees (Fa. Hevert): Kombination mit Hopfen und Passionsblume, $3 \times$ tgl. zur Beruhigung 2 Tbl. beziehungsweise bei Schlafstörungen vor dem Einschlafen 1-2 Tbl. einnehmen

- Calmedoron ${ }^{\circledR}$ Tropfen (Fa. Weleda): Kombination mit Hafer, Passionsblume, Hopfen und Coffea tosta D60, vor dem Schlafengehen 20-40 Tr. in etwas Wasser einnehmen

- Ardeysedon ${ }^{\circledR}$ Tbl. (Fa. Ardeypharm): Kombination mit Hopfen, 1- bis 3-mal tgl. 2 Tbl. einnehmen 


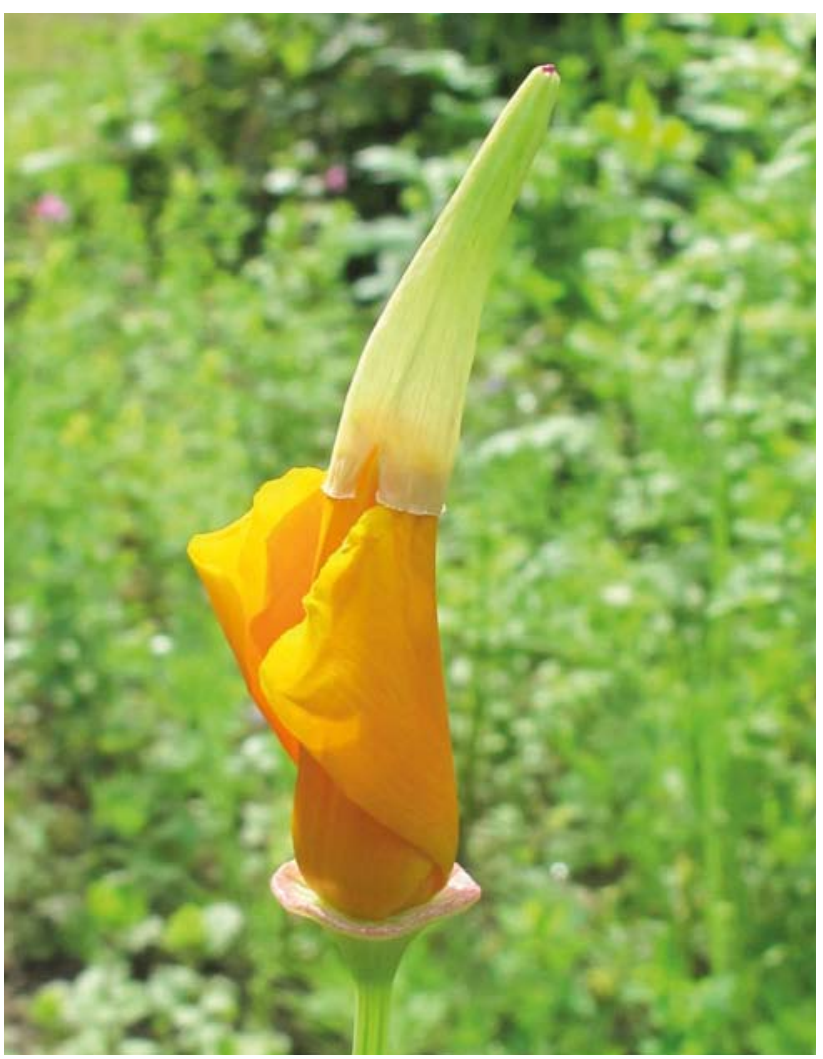

Abb. 2 Kalifornischer Mohn: Die Blütenknospe ähnelt kurz vor dem Aufgehen einer Schlafmütze, die in der Signaturenlehre als Qualität der Geborgenheit gedeutet werden kann. Das kräftige Orange erinnert an Lebens- und Tatkraft.

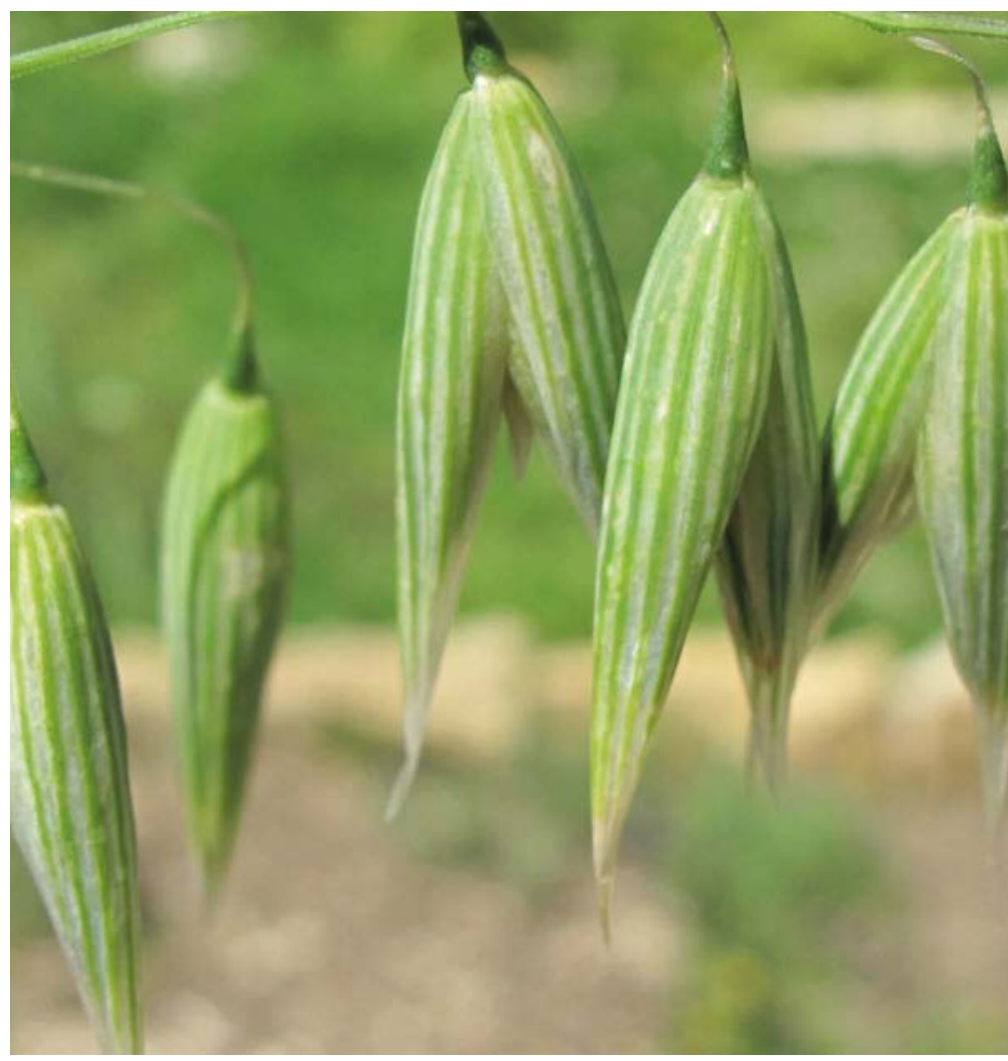

Abb. 3 Hafer: grün, weiß, grün - die rhythmisch angeordnete Färbung der Spelzen kann als die Fähigkeit gedeutet werden, ordnende Rhythmen ins Leben zu bringen.

\section{Kalifornischer Mohn (Eschscholzia californica)}

Bei älteren Menschen und bei kleinen Kindern kann der Kalifornische Mohn, auch Goldmohn genannt, wahre Wunder vollbringen.

\section{Merke: Die ungeschriebene Regel lautet, dass bei kleinen Kindern und im Alter bei Schlafstörungen oft die gleichen Pflanzen wirken.}

Signatur: Schlafmütze mit Leuchtkraft

Der Kalifornische Mohn weist in der Signatur seiner Blütenknospe kurz vor dem Aufgehen einen deutlichen Bezug zum Schlafen auf: Seine beiden Kelchblätter umschließen die Blüte in Form einer Schlafmütze und fallen beim Erblühen ab. Dies hat der Pflanze den Volksnamen „Schlafmützchen“ gegeben. Die Blüte ist von einem kräftig leuchtenden Orange.

\section{Wirkung: Geborgenheit und Lebensfreude}

So wie die Schlafmützen-Signatur Schutz und Geborgenheit vermittelt, so wird auch die Pflanze in ihrer Wirkung als Schlafmittel beschrieben. Man fühle sich so, als ob man in den Arm genommen werde und sich der Ruhe des Schlafs hingeben dürfe. Nicht nur Kindern, sondern auch älteren und pflegebedürftigen Menschen, die sich oft einsam und verlassen fühlen, kann diese Wirkung in einer unruhigen Nacht Trost spenden.

Die orangeleuchtenden Blütenblätter können als kraftvolles Zeichen der Lebensfreude und der Energie für den Tag gedeutet werden - etwas, das älteren Menschen vermehrt verloren geht.

\section{Kalifornischer-Mohn-Präparate}

" getrocknetes Kraut für die Teezubereitung

- Somcupin ${ }^{\circledR}$ spag. Peka Tropfen (Fa. Pekana): Urtinktur in Kombination mit Hafer und diversen homöopathischen Mitteln, 1- bis 3-mal tgl. je 5 Tr. in etwas Wasser einnehmen

- Passiflora Pentarkan ${ }^{\circledR}$ S Tropfen (Fa. DHU): Urtinktur in Kombination mit Hopfen, Passionsblume und Zincum D6, 1- bis 3-mal tgl. je 5 Tr. in etwas Wasser einnehmen

\section{Hafer (Avena sativa)}

Hafer ist den meisten Heilpflanzenkundigen nicht als Schlafpflanze geläufig und kann bei Schlaflosigkeit dennoch sehr hilfreich sein. An sich gilt Hafer als Mittel zur Nervenstärkung. Nicht umsonst sagt man, Hafer sei „reine Nervennahrung“, oder „den sticht der Hafer“, wenn ein Pferd angeblich zu viel Hafer zum Fressen bekam und übermütig wird.

\section{Signatur: Elastisch und aufrecht}

Schaut man sich die Signatur des Hafers an, erkennt man Qualitäten wie Beweglichkeit und Elastizität. Fegt ein Sommergewitter über das Land, liegen meist große Teile von Weizenfeldern darnieder. Hafer hingegen beugt sich mit seinem elastischen Stängel jedem Windstoß. Und wenn der Sturm vorüber ist, steht er unbeirrt wieder aufrecht da. Wirft man einen genaueren Blick auf die Spelzen, die das Haferkorn umgeben, erkennt man sich rhythmisch abwechselnde weiße und grüne Streifen. 


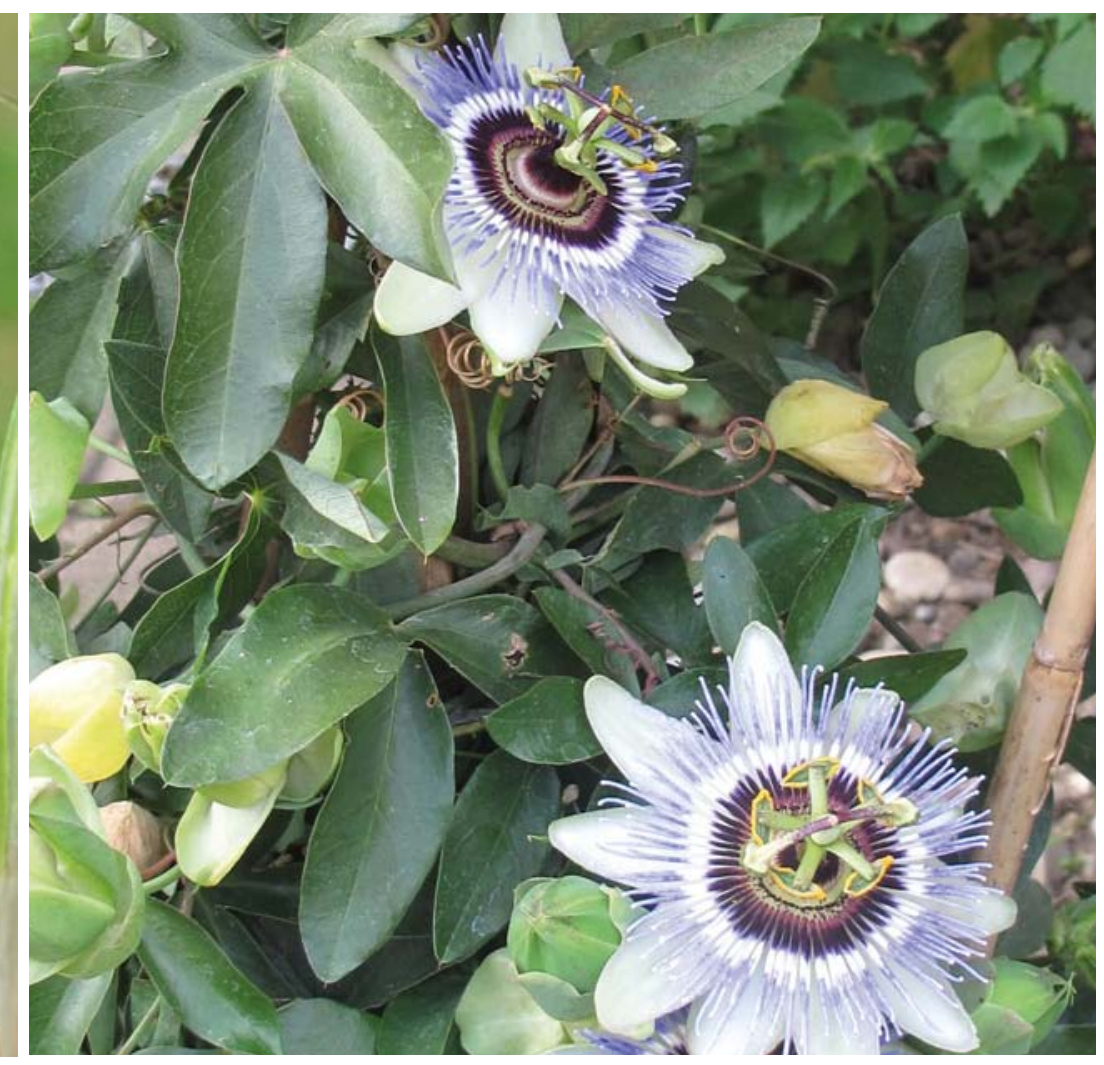

Abb. 4 Passionsblume: Beruhigung und Zentrierung sind die zentralen Themen ihrer Signatur. Die Blüte wird von außen nach innen, von der Nebenkrone bis zu den Staubblättern und den Griffeln, immer strukturierter und symmetrischer, also ruhiger. Die entsprechende Wirkung der Pflanze: Beruhigung in unruhigen Zeiten.

\section{Wirkung: Resilienz und Rhythmisierung}

Hafer ist das Mittel der Wahl, wenn Schlafstörungen und nervöse Erschöpfungszustände aufgrund zu hoher Belastung und Überforderung entstehen. Er schenkt seelische Elastizität und Widerstandsfähigkeit im Sinne der Resilienz.

Die rhythmische Farbigkeit der Spelzen wiederum weist auf die Fähigkeit des Hafers hin, ordnende Rhythmen ins Leben zu bringen. Säuglinge, Kleinkinder und Kinder, deren Tag- und Nachtrhythmus nicht gefestigt ist, die in der Nacht hellwach sind und den Eltern den letzten Nerv rauben, profitieren besonders vom Hafer. Aber auch in Zeiten der Pubertät kann die rhythmisierende Kraft des Hafers sehr hilfreich sein, um den Wechsel vom Kind zum jungen Erwachsenen zu begleiten.

\section{Hafer-Präparate}

- getrocknetes Haferkraut für die Teezubereitung

- Avena sativa Urtinktur (Fa. Ceres): 1- bis 3-mal tgl. 2-5 Tr. beziehungs- weise vor dem Schlafengehen 3-7 Tr. in etwas Wasser einnehmen

- Calmedoron ${ }^{\circledR}$ Tr. (Fa. Weleda, früher Avena comp.): Kombination mit Baldrian, Passionsblume, Hopfen und Coffea tosta D60; vor dem Schlafengehen 20-40 Tr. in etwas Wasser einnehmen

- Avena sativa Similiaplex ${ }^{\circledR}$ Tropfen (Fa. Pascoe): Urtinktur in Kombination mit diversen homöopathischen Mitteln; 1- bis 3-mal tgl. je 5 Tr. in etwas Wasser einnehmen

- Avena sativa Urtinktur (Fa. Hanosan): 1- bis 3-mal tgl. je 5 Tr. in etwas Wasser einnehmen

- Nervoid ${ }^{\circledR}$ N Tropfen (Fa. Hanosan): Urtinktur mit Traubensilberkerze, Passionsblume, Baldrian und diversen homöopathischen Mitteln; 1- bis 3-mal tgl. je 5 Tr. in etwas Wasser einnehmen

- Cefaneuro ${ }^{\circledR}$ Tabletten (Fa. Cefak): Kombination mit Passionsblume und Ignatia D4 und Gelsemium D4; 1- bis 3-mal tgl. 1 Tbl. im Mund zergehen lassen

- Pasconal ${ }^{\circledR}$ Nerventropfen (Fa. Pascoe): Kombination mit Baldrian, Strychnos 


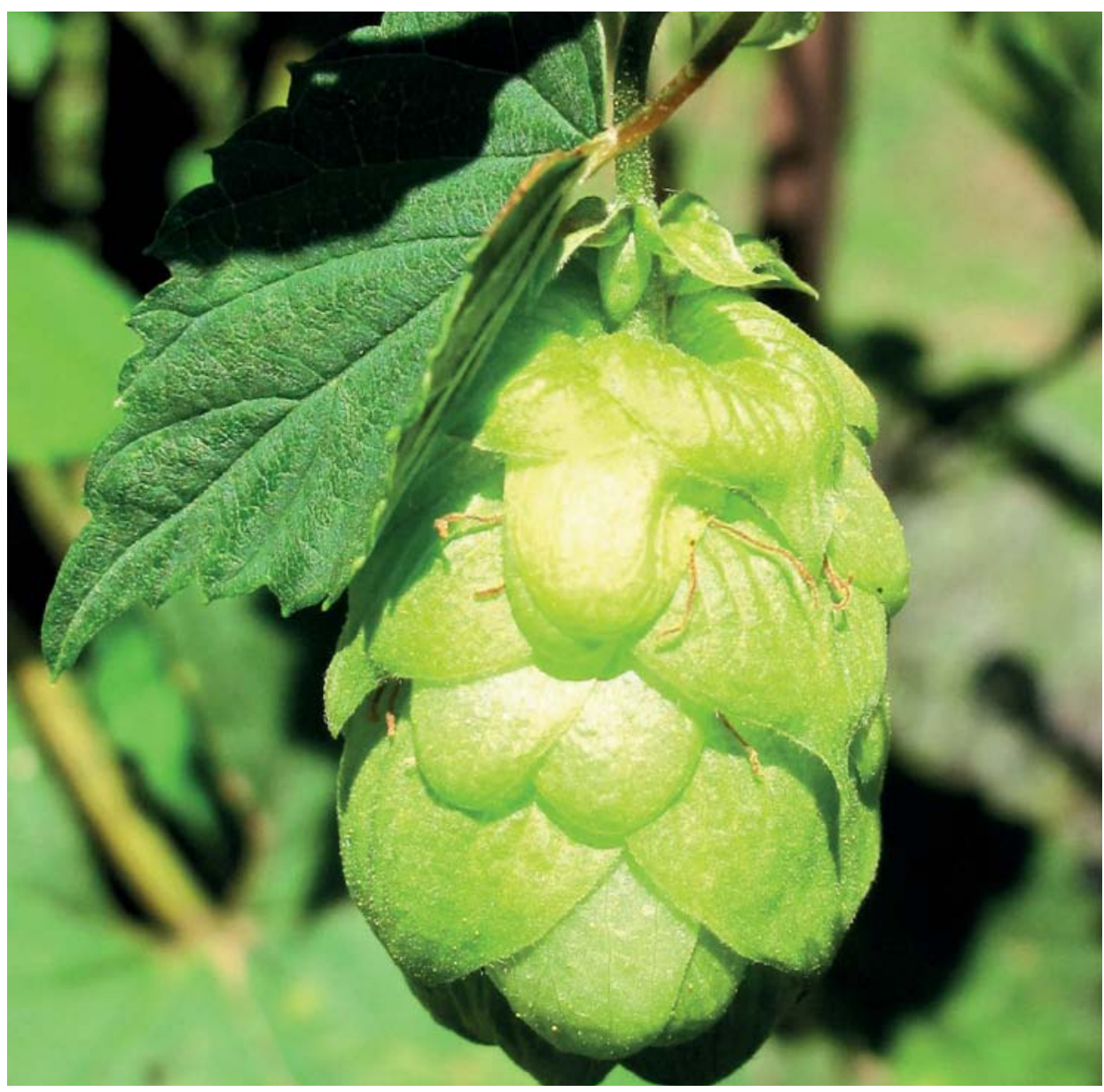

Abb. 5 Hopfen: Er ist berühmt dafür, dass er bei der Ernte auf Frauen anregend, auf Männer im wahrsten Sinne einschläfernd wirkt. Hopfen-Patienten sind hellwach in der Nacht und todmüde am Tage.

D4 und Tarantula D5; 1 - bis 3-mal tgl. je 5-10 Tr. in etwas Wasser einnehmen

\section{Passionsblume (Passiflora incarnata)}

Die Passionsblume ist dann angezeigt, wenn Schlafprobleme mit Sorgen, Kummer und Ängsten vergesellschaftet sind. Das können Albträume bei Kindern sein, die schreiend aus dem Schlaf hochschrecken und nur noch mit Licht, offener Schlafzimmertüre oder im Elternbett schlafen wollen. Genauso kann es sich bei der Ursache um mehr oder weniger definierte Ängste, Kummer und Sorgen handeln wie Verlustängste, Angst vor Kündigung, finanzielle Sorgen, Angst vor dem Verlassen werden, Angst vor dem Alter. Hier sind eher Erwachsene die Zielgruppe.
Signatur: Vom Chaos in die Konzentration

Einmal mehr zeigt sich in der Signatur einer Schlafpflanze ein rhythmisches Prinzip. Die Blüten der Passionsblume besitzen viele konzentrische Kreise, die den Blick unwillkürlich auf die Mitte, auf ihr Zentrum lenken (siehe Abb. 4). Auffallend dabei sind die ungefähr 100 dünnen, fast faserig anmutenden, violettweiß marmorierten Blütenblätter der sogenannten Nebenkrone. Nach außen hin sind diese oft etwas schlangenlinienförmig gebogen und strahlen dadurch ziemlich viel Unruhe aus. Im Innern der Blüte folgen 5 Staubblätter und der Fruchtknoten mit 3 Griffeln. Von außen nach innen wirkt die Blüte also immer ruhiger, zentrierter, die unruhigen Wellen lösen sich auf.

Wirkung: Stille in unruhigen Zeiten Der in der Blüte von außen nach innen immer ruhiger werdende Rhythmus von
Form und Farbe ist ein Hinweis auf die beruhigende Wirkung der Passionsblume in bewegten Zeiten. Ähnlich der Ruhe, die sich nach einem Steinwurf ins Wasser und den vielen daraus entstandenen Wellenringen wiedereinstellt.

\section{Passionsblume-Präparate}

- getrocknetes Passionsblumenkraut für die Teezubereitung

- Passiflora incarnata Urtinktur (Fa. Ceres): 2- bis 4-mal tgl. 2-5 Tr. oder vor dem Schlafengehen 5-10 Tr. in etwas Wasser einnehmen

- Passiflora Kinderzäpfchen (Fa. Wala): in Kombination mit Baldrian und Hopfen, abends 1 Zäpfchen in den Mastdarm einführen

- Passiflora Nerventonikum (Fa. Wala): in Kombination mit Hopfen, Weißdorn, Hafer, Baldrian, Hypericum D2 und Salix D2, bei Schlafstörungen vor dem Schlafengehen 1-3 TL Sirup, bei Unruhe $3 \times$ tgl. 2 TL einnehmen

- Passiflora Curarina ${ }^{\circledR}$ Tropfen (Fa. Harras Pharma Curarina): $3 \times \operatorname{tgl} .2 \mathrm{ml}$ einnehmen

- Passidon $^{\circledR}$ (Fa. Ardeypharm): bei nervöser Unruhe $2 \times \operatorname{tgl}$. 2 Kps. einnehmen

\section{Hopfen (Humulus lupulus)}

Der Hopfen ist eine Kletterpflanze, die hoch hinaus wachsen will und ihre ganze Kraft in dieses Ziel steckt. Die weiblichen Hopfenzapfen werden für Arzneimittel, aber auch für Bier verwendet.

\section{Signatur: Lange heiter versus schnell müde}

Um die Signatur des Hopfens in Bezug auf Schlafstörungen zu verstehen, bedarf es eines kleinen Ausflugs in die Welt der Hopfenernte. Die Erfahrung besagt, dass man bei der Ernte der duftenden Zapfen stets heiter und fröhlich gestimmt sei. Bei den rauschenden Tanzfesten, die nicht selten im Anschluss an eine Hopfenernte stattfinden, seien Frauen deutlich länger aktiv als die Männer. Diese würden schneller müde und irgendwann von einer einschläfernden Schwere eingeholt werden. Dabei ist an die östrogen- 
ähnliche Wirkung der Hopfenzapfen zu denken, die Frauen munter und fröhlich macht und bei den Männern eher als Anaphrodisiakum wirkt.

\section{Wirkung: Ordnung im Rhythmus- chaos}

Diese beschriebene Diskrepanz von Wachheit und Müdigkeit zeigt sich bei Hopfen-Patienten sozusagen in Personalunion. Sie können sowohl voller hellwachem Tatendrang als auch von erdenschwerer Schläfrigkeit geplagt sein. Das größte Problem dabei ist, dass sie beides jeweils zur falschen Zeit sind: nachts sind sie hellwach und tagsüber hundemüde.

Der Hopfen vermag es, den umgedrehten Tag-Nacht-Rhythmus wieder in die richtige Bahn zu lenken und nachts für erholsame Ruhe zu sorgen. Die Bitterstoffe des Hopfens eignen sich gut bei Verdauungsstörungen, auch in der Nacht, wenn nach einem fetten Abendessen der Magen-Darm-Trakt rumort und nicht die für den Schlaf notwendige Ruhe gibt.

Hopfen-Präparate (Auswahl)

" getrocknete Hopfenzapfen für die Teezubereitung

- Lupulus Urtinktur (Fa. Ceres): 1- bis 3-mal tgl. 2-5 Tr. in etwas Wasser einnehmen

- Humulus lupulus Urtinktur (Fa. Weleda): 1- bis 3-mal tgl. 5-10 Tr. in etwas Wasser einnehmen

- Tondinel ${ }^{\circledR} \mathrm{H}$ Tropfen (Fa. Pflüger): in Kombination mit diversen homöopathischen Mitteln: 1- bis 3-mal tgl. 5-10 Tr. in etwas Wasser einnehmen

\section{Nicht zu vergessen:}

\section{Seelen- und Herzpflanzen}

Selbstverständlich verfügt die Schatzkiste der Natur über weitere Heilpflanzen, die man bei Schlafproblemen einsetzen kann, auch wenn sie keinen direkten Bezug zu Einschlaf- und Durchschlafstörungen haben. Als Beispiele seien an dieser Stelle der Lavendel und der Weißdorn genannt.

Wenn Kummer und Sorgen übermächtig werden und einen nicht mehr schlafen lassen, dann kann der Lavendel diese im übertragen Sinne von der Seele waschen. Daher stammt womöglich auch sein Name: „Lavare“ kommt aus dem Lateinischen und bedeutet „waschen“.

Der Weißdorn als wichtigste Herzpflanze, im Volksmund interessanterweise auch Schlafdorn genannt, hilft bei funktionellen Herzbeschwerden wie Herzklopfen, -drücken, -stolpern und -schmerzen. Im übertragenen Sinne gilt das auch für Herzschmerzen wie Liebeskummer oder Sorgen um die Liebsten. Diese Symptome treten gerne in der Nacht auf und hindern die Betroffenen dann am Schlafen. Hier kann man an den Weißdorn alleine oder in Kombination mit dem Herzgespann einnehmen.

Alle Abbildungen: ㄷ Cornelia Stern

Dieser Artikel ist online zu finden:

http://dx.doi.org/10.1055/a-0832-4223

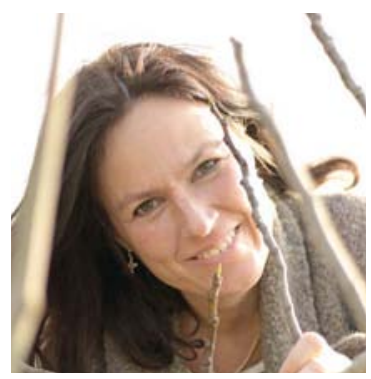

\section{Cormelia Stern}

Mitinhaberin der Freiburger Heilpflanzenschule

79111 Freiburg im Breisgau

E-Mail: cornelia.stern@heilpflanzenschule.de

Internet: www.heilpflanzenschule.de

Cornelia Stern ist Apothekerin mit Spezialisierung in Naturheilkunde. Zusammen mit ihrem Mann leitet sie die Freiburger Heilpflanzenschule und ist als Dozentin für Phytotherapie, Spagyrik, Gemmotherapie und Klassische Homöopathie tätig. Zudem ist sie Autorin der Bücher „Gemmotherapie“, „Die Heilkraft der Pflanzenknospen“ und Mitherausgeberin der $\mathrm{DHZ}$. 